\title{
ARRANCAME LA VIDA, DE ANGELES MASTRETTA: EL UNIVERSO DESDE LA INTIMIDAD
}

\author{
POR \\ ALICIA LLARENA \\ Universidad de Las Palmas de Gran Canaria
}

Sé que te caben muchas mujeres en el cuerpo y que yo sólo conocí a unas cuantas.

(Arráncame la vida).

\section{UN CONTEXTO: ESCRITURA Y "POSMODERNISMO"}

Todo acercamiento a la narrativa de los años 80 muestra en seguida la heterogeneidad de una escritura que se esparce en autores, estilos y tendencias de las más variadas condiciones. Pero, si es cierto que la diversificación en la última narrativa es evidente, también lo es que algunos de sus rasgos y otras tantas recurrencias temáticas pueden permitirnos, cuando menos, penetrar en la década cual eficaces hilos de Ariadna dentro de un laberinto -como todo lo reciente-provisional. Algunos críticos, a los que en seguida vamos a referirnos, han intentado apuntar con ellos las líneas básicas de esta última escritura, y han conseguido así delimitar parcelas cumpliendo el requisito indispensable de la clasificación: el ordenamiento de la realidad. A este ejercicio crítico debe sumarse, además, la discusión cada vez más abundante sobre qué sea y cuáles son los gestos destacables de ese inquieto fenómeno al que llamamos "la posmodernidad". A la luz de ese cuestionamiento sobre los nuevos modos de lo contemporáneo, ciertos autores han logrado esclarecer también los síntomas generales de la última narrativa. Con esta doble referencia crítica y teórica, la aproximación a la escritura de Angeles Mastretta (México, 1949) podría revelar sin duda algunas claves que expliquen su excelente y vertiginosa difusión: meses después de publicar Arráncame la vida ya eran seis las ediciones de su novela, y más de diez los idiomas en los que su opera prima puede leerse hasta el momento. Por supuesto, como cabría esperarse, el éxito temprano de Angeles Mastretta ha despertado encontradas posiciones entre la crítica, y junto a aquellos que comentan su visión renovadora no falta quienes la entienden -0 la sospechan-como un puro acto comercial'; entre ambos extremos, y sobre

1 "Es una buena novela - dice Christopher Domínguez Michael- tersa en sus procedimientos y sencilla en sus pretensiones. Pero su increíble éxito internacional 
todo frente a este último, planteamientos como el de Jorge Ruffinelli encierran a nuestro juicio la postura quizá más coherente o ejemplar:

... deberíamos reflexionar sobre los cambios actuales, vertiginosos, de los paradigmas literarios y su relación con los cambios sociales, ante todo con el imaginario social. (...) Quiérase o no, la literatura ha cambiado, ha venido cambiando en dirección a lo que es hoy y dejará de ser mañana. Lo que se impone es preguntarse también qué es lo que la ha estado cambiando, y "hacia donde y para quén sus cambios (42).

En pos de la anotación de esos "vertiginosos" cambios, las incursiones teóricas realizadas al respecto coinciden en señalar la relación estrecha de la última narrativa del continente americano y el "posmodernismo". Sánchez Vázquez esboza esa conducta reciente en torno a ciertos núcleos que otros aplican de igual modo a la escritura, y de los que destacamos dos: la "desdramatización del fin" (nueva moral de la muerte y consiguiente reconciliación con la realidad), y el eclecticismo (es decir, la reivindicación de lo fragmentario, de lo local o regional frente a las narraciones totalizantes modernas). Este mismo enfoque, destilado en torno a la narrativa latinoamericana de los años 80 , es el que sigue Ruffinelli para mostrar su personal balance de la década. Muchos de los rasgos que él apunta pueden verse, sin ir más lejos, entre las líneas de Angeles Mastretta, "producto" literario -como cualquier otro- de su momento histórico. En los últimos tiempos "se pulveriza la noción de centro, orden y jerarquía, y se inicia un novísimo trabajo sobre los márgenes, las fronteras, las periferias, las 'minorías" -comenta Ruffinelli- y se "celebra la de-sacralización de los productos artísticos y su sustitución por el consumismo democratizador de la 'cultura de masas', la mercantilización del conocimiento y el arte; el culto al 'pastiche' que niega al individualismo y a la originalidad" (32).

Algunos de los narradores del post-boom americanocoinciden también, desde el seno mismo de sus escrituras, en la advertencia sobre los nuevos presupuestos literarios de las últimas décadas, indicando que "ha ocupado el primer plano una visión fragmentaria, crítica, irónica, humorística", y es frecuente "laintromisión de la poesia, de la libertad verbal, de los lenguajes coloquiales" porque "se insiste en un lenguaje libre, flexible, (...) y se busca cierta simplificación estructural", comoapunta Jorge Edwards (68), o que "se ha perdido - a dios gracias-aquella insoportable necesidad de escribir 'La-Gran-Novela-Latinoamericana' (así, con mayúsculas) que pareció desvelar a los maestros del boom y de poco antes", a

corresponde más a las nada caprichosas leyes del comercio que a su profundidad literaria"; "Los críticos - continúa más tarde- que creyeron encontrar en Arráncame la vida la llamada de un nuevo realismo se equivocaron candorosamente. Se trata de un realismo rosa que no se atreve a decir su nombre, plagado de guiños convencionales y técnicas manidas" (498-499). 
juicio de Mempo Giardinelli (23). Es este último quien señala también el "retorno a la espontaneidad"2 que rige el reciente ejercicio narrativo, cuestión que observa a un tiempoSkármeta para diferenciar la práctica "más espontánea", con "más humor", y "menos consciente de sí misma" que los separa del boom: "donde ellos se distancian abarcadores, - dice - nosotros nos acercamos a la cotidianeidad con la obsesión de un miope"s. Todos estos juicios remiten al profundo "eclecticismo" y a la no menos trascendente "reconciliación con la realidad" que fluye en la conciencia y la conducta "posmoderna", como ya se indicó. Ámbos rasgos, ciertamente importantes a la hora de hacer valoraciones en torno a la última narrativa del continente, hacen surgir de inmediato nuevos e interesantes puntos de vista, hasta entonces marginales, en el discurso literario americano (marginalidad cuyo abanico de acción y cuyos grados y matices suponen, parafreseando a Giardinelli, una inmejorable oportunidad para el imaginario de cualquier autor $)^{4}$. Sobre ese horizonte múltiple, sobre esa permanente "descentralización" de la narrativa actual, se asienta esta otra "cultura de la periferia", términos en los que Nelson Osorio se aproxima a la creación reciente de América Latina. Entre las señas de identidad que él destaca se perciben las variadas formas, temas, estilos y maneras con que todas estas visiones de la critica se materializan en la novela o en el relato: escenarios radicales de periférica urbanidad, temática cotidiana y a veces irreverente, lenguaje coloquial, directo y hasta soez; estructuras flexibles, ligeras, contaminadas de otros discursos paralelos (la canción, el cine, lo rosa, el folletín) y perspectivas novelescas que no formaban parte del presupuesto literario habitual (la homosexualidad o la mujer) han sido, con preferencia, sus instrumentos 5 . Y es tal vez ese centro orientador de toda actividad narrativa lo que nos interesa de un modo especial.

Precisamente, la irrupción en el panorama narrativo actual de algunas de esas perspectivas periféricas, descentralizadas o marginales, nos lleva de la mano hacia el universo particular de Angeles Mastretta, novelista que representa, entre muchas otras, una de las tendencias generales en torno a las cuales Ruffinelli clarifica la última narrativa. "Lo popular", "el testimonio" y "el

3 Jorge Ruffinelli, loc. cit., 37.

a Jorge Ruffinelli.

4 “... Ia narrativa mexicana ha estado demasiado sometida al imperio de dos o tres estilos y temáticas, y por ello en los proximos años, en los 90 , deberá empezar a ocuparse de otros temas acaso más vinculados con la discusión finisecular sobre la democracia, así como de los nuevos aspectos de la crisis social y económica y también cultural que viven nuestros países, crisis que no sólo es un idóneo material para descripciones naturistas, sino, mejor, una asombrosa oportunidad para el imaginario de cualquier au tor", Giardinelli (22).

5 "Ficción de oralidad y cultura de la periferia en la narrativa hispanoamericana actual", conferencia impartida dentro del Ciclo "Literatura moderna en América Latina: ficción y marginalidad", celebrado en la Universidad de Las Palmas de Gran Canaria, febrero de 1990. 
feminismo" son, a su juicio, los centros en los que convergen las diferencias de la ficción continental, y es en el último de los tres, sin duda alguna, donde se afianza la escritura de nuestra narradora. Pero insistiremos, antes de entrar en su universo, y coincidiendo con Ruffinelli, que esa voz desde el interior de la mujer, desde la intimidad de una conciencia periférica, marginada, desde "el feminismo", no debe entenderse como la práctica de un "feminismo al uso antiguo (...), ni siquiera la pregunta por una escritura especifica, ni la exhumación de escritoras del pasado, sino ante todo el feminismo como nueva perspectiva de análisis que denuncia y cuestiona radicalmente las falsas hegemonías, el poder de una cultura patriarcal y homocén trica ${ }^{\$ 6}$ (38-39). La obra de Angeles Mastretta se incorpora, así, a una larga serie de narradoras que interpretan el mundo a través de esa "nueva perspectiva", y que encuentran, en el marco de la posmodernidad, razones más que evidentes para su constante manifestación. En un océano predominantemente masculino, la voz marginal de la mujer se

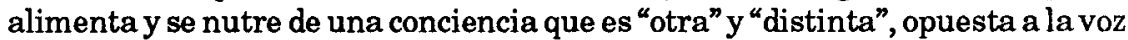
convencional. No es poca, en este sentido, la trascendencia de la observación de Mempo Giardinelli cuando señala que las mujeres "curiosamente, al contrario de lo que pasó en otros países, en México -país de machismo proverbialsiempre tuvieron un lugar en la narrativa", desde la Revolución con los cuentos de Campobello, Galeana y Cármen Báez y, sobre todo, a partir del 68 "hasta llegar a la hoy popularísima Angeles Mastretta, autora de la sabrosa Arráncame la vida, uno de los más estruendosos best-sellers mexicanos" ${ }^{m}$. No deja de ser sintomático que en el país de mirada homocéntrica la escritura feminista haya crecido en la medida justa en que ha sido relegada (proporción inversa sin duda interesante para una interpretación "posmodernista"), y que su último éxito literario y comercial coincida con un texto que reivindica, como pocos, su parcela de poder y de visión. La vida de un hombre público desde los secretos que acumula la intimidad, o la "historia de la esposa de un célebre general posrevolucionario vista desde la alcoba”, como la define de un trazo Domínguez Michael $^{8}$, y el conjunto de mujeres con historia que compone Mujeres de ojos grandes (la última publicación de la autora mexicana, en 1991), convierten por ello a nuestra narradora en un caso paradigmático y especial. Serán, pues, la

\footnotetext{
${ }^{6}$ El subrayado es nuestro.

${ }^{7} \mathrm{Y}$ desde, por lo menos, el 68, hay que anotar una interesantísima variedad de escritoras: la mencionada Poniatowska, Luisa Josefina Hernández, Josefina Vicens, Inés Arredondo, Amparo Dávila, Guadalupe Amor, Julieta Campos, Margo Glantz, Esther Seligson, Sara Sefchovich, Mónica Mansour, Ethel Krauze, Jacobs, Puga, Mendoza y muchas otras más", Mempo Giardinelli (25).

8 "La pertinencia de la novela —añade-radica en el suave desarrollo de la pasión de una mujer, su paseo por las habitaciones de la tradición y el hogar, la ropa y el comercio carnal, el matrimonio y el adulterio", aunque también a su juicio "la feminidad en nuestra novela tiene mucho más que decirn (499).
} 
perspectiva femenina, el discurso profundamente emocional, la reivindicación de las pasiones contra el tedio o contra el olvido, el humor y la ironía con que sus mujeres-personajes libran su batalla en un contexto familiar y cotidiano, la agilidad narrativa con que logra definir cualquier carácter y mostrar las situaciones de este universo particular, desde su propio vientre, comoindicábamos arriba, los rasgos que, entre otros, el lector advertirá en un primer acercamiento a sus ficciones.

\section{UNA LECTURA: FEMINISMO E INTIMIDAD}

La voz de Catalina, desde el comienzo de Arráncame la vida, es un indicio que marca pronto la dinámica posterior de la novela, y la que anuncia el progresivo desarrollo, la explosión, de su resignada intimidad: "Tenía quince años y muchas ganas de que me pasaran cosas" (11) - comenta. Sin embargo, su joven apetencia da lugar a una iniciación que percibe en seguida los límites de su propia periferia, terreno que crece a golpes eficaces, narrativos y existenciales: su general "hablaba conmigo como con las paredes, sin esperar que le contestara, sin pedir mi opinión" (13) - dice-, aunque la torpe iniciación -incluida la sexual-a la que se somete, permita por otro lado "que más que pensar en él" quede "obsesionada con sentir" (14). Tal vez sea éste el primer gesto de emancipación al que asistimos en el transcurso de la novela, y la primera e inquietante aceptación de la individualidad del personaje: su curiosidad no recibió la calma hasta que la gitana del barrio de La Luz le explicara ese secreto irreductible a través del cual "todo lo importante estaba ahí, por ahí se miraba, por ahí se oía, por ahí se pensaba (...) Las piernas se me pusieron tiesas como si quisieran desprenderse. Y sí, ahí estaba todo" (16). En pocas páginas, con natural agilidad narrativa, Angeles Mastretta nos sumerge en la intimidad del personaje, y nos fabrica un general cuya despiadada corrupción social no es más intensa que el despotismo con el que trata a sus mujeres. Pero la más interesante antítesis o dualidad de la novela tiene su origen, precisamente, en esa relación curiosa entre "inocencia" y "autoridad", entre el poder central y sus desafortunados - en este caso femeninosextrarradios; en la medida en que el general se pasó años "viéndome a veces como una carga, a veces como algo que se compra y se guarda en el cajón" (35), y ella permanecía "guardada como un juguete con el que se platicaba de tonterías, al que se cogía tres veces a la semana y hacía feliz con rascarle la espalda y llevar al zócalo los domingos" (37), la mujer experimenta la necesidad "de que algo le pase, lo que sea, menos las mañanas iguales"(35). Aterrorizada con la idea de convertirse en madre, su primer embarazo recibe una de aquellas cosas que reclamó su despierta obsesión por disfrutar la fortaleza de lo íntimo: Pablo, un amigo del colegio, "se encargó de quitarme las ansias esos tres últimos meses de embarazo, y yo me encargué de quitarle la virginidad que todavía no dejaba en ningún burdel" (41). Pero la vida de esta mujer no acaba de 
encauzarse bien hasta después, $y$ en un zigzagueo permanente entre la vivencia de su instinto, y la convención o la racionalidad, se acoge a la comodidad de lo segundo y se resigna. Innumerables hijos de su esposo se añadirán a los suyos propios, y las variadísimas amantes, a las que envidia "porque ellas sólo conocían la parte inteligente y simpática de Andrés, estaban siempre arregladas cuando llegaba a verlas, y él no les notó nunca los malos humores ni el aliento en las madrugadas" (71), le roban con frecuencia a su general. Su ignorancia, natural y lógica en la adolescencia, se convierte entonces en un premeditado modo de estar, ficticia tragicomedia en cuyo reparto se autoasigna el principal papel; de este modo, convencida de que "era la mamá de sus hijos, la dueña de su casa, su señora, su criada, su costumbre, su burla", también lo está de que "lo tenía que seguir siendo por más que a veces me quisiera ir a un país donde él no existiera" (72). El planteamiento narrativo de esta contradicción se sustenta, básicamente, en una perspectiva novelesca que, desde el testimonio directo de la primera persona afianza el individualismo y la subjetividad. Cabe señalar también que la mujer adulta y liberada que Catalina es cuando nos confía los secretos de su vida, añade a la narración el punto necesario de distanciamiento que explica toda flexibilidad en la rememoración de su pasado, toda ironía. Por otra parte, el tono coloquial de Arráncame la vida nos permite ahondar en las directas impresiones que transmite su narradora, sobre todo cuando en la transcripción de los diversos diálogos las voces implicadas se desnudan y retratan en su espontaneidad. La agilidad narrativa de Angeles Mastretta, su eficacia y economización de la estructura y del discurso en general, tienden a imponer un ritmo oscilante y vertiginoso en la novela, que conduce de modo inevitable a su climax, ese momento en el que asistimos al despertar del personaje: el general "me trepó el vestido y yo apreté las piernas", “... yo seguí con las piernas cerradas, bien cerradas por primera vez" (98-99). Es entonces cuando ese feminismo "como nueva perspectiva de análisis que denuncia y cuestiona radicalmente las falsas hegemonias" -como dijera Ruffinelli- se derrama en múltiples escenas y elementos de la historia. Desde estas palabras, las últimas del capítulo VII, a las que inician el siguiente, la diferencia es absoluta, y la eficacia providencial: "Desde que vi a Fernando Arizmendi me dieron ganas de meterme en la cama con él" (101), nos cuenta Catalina, anunciando con ello el alumbramiento de la intimidad. A partir de aquí esas regiones "marginales" y "sensibles" de lo femenino se manifiestan contra la apagada capacidad de amar, soñar, o de sentir, que reclama en adelante con sus hechos: "Ya te salió lo mujer. Está usted hablando de su inteligencia y luego le sale lo sensiblero", es lo último que oyó del general antes de volcarse con plenitud hacia su propio interior, sin renunciar a los diversos gestos de la "cultura periférica" aun a riesgo de confundirse, para muchos, con la heroína de un folletín. Más que como inútil "realismo rosa", gratuito pastiche o conjunto de "guiños convencionales", como se ha visto en esta novela, el registro emocional

- Vid. nota núm. 1. 
no juega a la fácil conexión con el lector, sino a la reivindicación visible, y a veces cómica, de ese otro modo de orientarse en la realidad. Esos últimos reproches, además, corren el riesgo de parecerse demasiado a las palabras del general, relegando la otredad, "lo sensible", "lo mujer", a un histórico y cultural segundo plano. Puede que las numerosas quejas sobre el tedioso papel de la mujer en Arráncame la vida hayan confundido a quienes la interpretan como un acto de caduco feminismo; y es natural, si esa lectura prejuiciosa y simple se detiene sólo en el cansancio de Catalina con respecto a "sus labores", su maternidad o su matrimonio. Pero, al margen de todo ello, lo esencial no es su progresiva aversión hacia las normas sociales, ni la declaración de la igualdad, sino al contrario, la aceptación de esas regiones íntimas, diferentes y auténticas, que el general veía -temía, quizás- como lo único inasible, incontrolable, desconocido, en las múltiples mujeres que hay en su mujer.

Las referencias "contra-culturales", así pues, no cesan de florecer en un texto que se muestra como reunión de efectos destinados a provocar la súbita recepción de la sinceridad. Por ello, la presencia de Catalina entre un grupo de mujeres que se amontonan en el aeropuerto en busca de Tyrone Power - poco antes de ejercer, por cierto, mediante el voto, la "condición de mujer oficial" (137)_, los romances múltiples a los que se entrega sin que sepa "qué hacer con el deseo fuera de sitio que me estaba creciendo" (178), y las alusiones explícitas a "Temor", "La noche de anoche", "Cenizas", y la propia "Arráncame la vida" (canciones cuyos discursos sentimentales clásicos se suman a la novela en un momento de máximo derroche emocional), e incluso la breve aportación de una revista con consultorio amoroso, y el recuerdo "sagrado" de La dama de las camelias, antes de ser recetas fáciles son, si acaso, hipérboles que cumplen en la novela una función irónica y un papel ilustrador, tan importantes por su capacidad simbólica como las confesiones que, desde dentro, y salpicadas por el texto, rezuman autenticidad: "Toda la vida me la he pasado queriendo que me quieran" (173), "tuve la nefasta ocurrencia de disertar sobre las incomodidades, lastres y obligaciones espeluznantes de la maternidad. Quedé como una arpía. (...) Habia detestado alguna vez ser madre de mis hijos y de los ajenos, y estaba en mi derecho a decirlo" (273). Como síntesis del proceso por el cual Catalina se adhiere a un feminismo de sello más moderno, revelado en la actitud cotidiana y en la asunción y vivencia plena de su ser, las páginas finales de la novela no podían ser más radicales, abundantes en lugares comunes cuyo convencionalismo devora, a trazos simples, una convención mayor: "quiero ... una casa ... en la que mande yo, en la que nadie me pida, ni me ordene, ni me critique" (299), "sin tus piernas cruzándose a media noche en mi camino, sin tus ronquidos" (300), dice en providencial soliloquio junto al marido muerto; palabras a las que se añade, finalmente, la sinceridad de sus sensaciones tras el entierro del general, en las últimas líneas de la novela:

Quise sentir la pena de no ir a verlo nunca más. No pude. Me sentŕ libre. (...) Estaba sola, nadie me mandaba. Cuántas cosas haría, pensé bajo la lluvia a 
carcajadas. Sentada en el suelo, jugando con la tierra húmeda que rodeaba la tumba de Andrés. Divertida con mi futuro, casi feliz.

\section{UNA VALORACION: HEGEMONIA, PERIFERIA Y SINCERIDAD}

Desde ese futuro, justamente, nos habla Catalina. Desde esa risa que le procura a la mujer una vivencia plena, sin mediaciones, de la intimidad del ser. $Y$ es en ese instante último donde toma cuerpo una interpretación del código social de la novela entendido ya no como un convencional y cotidiano desarrollo del personaje central de Arráncame la vida, sino como una forma de expresar, de modo inevitable, con nervioso trazo y urgente economía, el relegado espacio de su otredad. La rememoración de su pasado desde la edad adulta y, aún más, desde su viudedad liberalizadora, contribuye a que su "paseo por la tradición y el hogar" no sea sólo un viaje por conocidas estampas de la vida femenina en busca de un nuevo -y equitativo- papel social, sino un recorrido por el arquetipo humano que subyace en el fondo de todas ellas. Es curioso que quienes han querido hallar una escritura especificamente femenina no han podido demostrar una subjetividad distinta fundada "casi exclusivamente en lo sexual", porque "parte de la diferenciación genérica que subyace en la dialéctica del discurso sexual está condicionada por componentes sociales ${ }^{n 10} \mathrm{o}$, si se quiere, porque en la relación escritura/sexualidad "el rol sexual es la mediación ... explicativa de toda diferencia que se sospeche originada en la sexualidad"11. Y tal vez porque la perspectiva femenina no pueda explicarse -como ninguna otra- sin esa múltiple mediación del código social, muchas de las acciones, gestos y actitudes de nuestro personaje parezcan a primera vista clichés convencionales, parte evidente de aquella curiosa relación entre "inocencia" y "autoridad" que Catalina recuerda desde una distancia objetivadora y feliz, signos tradicionales, en fin, que devienen en usual rebeldía emancipadora. Sin embargo, la "liberación" de Catalina opera en un sentido más amplio que la simple revisión de esta feminidad social con signo externo, conclusión que se extrae al comprobar que más que la exigencia a través del grito o del reproche de los privilegios y derechos homocéntricos, son sus acciones progresivas, su

\footnotetext{
${ }^{10}$ Marta Morello-Frosch, "Discurso er6tico y escritura femenina", Escritura y sexualidad en la literatura hispanoamericana, Madrid:Fundamentos, 1990,29. Aestas conclusiones Morello-Frosch añade que "Una escritura y lectura feminista debe tener en cuenta no sólo los impulsos rebeldes y autónomos del sujeto, sino también sus deseos socialmente condicionados de amor y dependencia, pues ambas tendencias contribuyen a la escision de la subjetividad en términos muy especfficos de factores sociales. Si no observamos estas contradicciones, podemos caer en una lectura que refuerza, como el romance popularizado por los medios masivos de comunicación, la ideología de dominación que queremos desvirtuar" (30); (el subrayado es nuestro).

${ }^{11}$ Alicia Puleo, "Perspectivas antropológicas de un problema de crítica literaria", Escritura y sexualidad en la literatura hispanoamericana, 19.
} 
propia actitud, la que habla y reclama su mejor reivindicación: lo distintivo de este "discurso periférico"; si profundizamos bien, no es tanto la explícita y evidente mostración del homocentrismo, la tópica reclamación de la igualdad social, sino más aún, el descubrimiento, la vivencia plena, y la consumación, de las diferencias.

Catalina no practica el adulterio para corresponder al adulterio masculino, ni para ejecutar con ello una reivindicación exclusivamente centrada en lo sexual, aunque sea ése uno de los modos en que se da respuesta a aquella obsesión temprana "por sentir" que siguió al primer contacto con Andrés. Los hombres de su vida fueron siempre producto de una extraña, ligera o arrebatada, "perturbación"; emancipación emocional que intenta superar el tedio y que despierta a menudo la envidia en la novela: cuandola gitana del barrio de la Luz al leer en la mano de Catalina comenta "Ay muchacha es que tú tienes muchos hombres aqui", Pepa confiesa que "a mí me gustaría tener una mano tan interesante como la tuya" (27); y en efecto años después ese deseo se materializa en un amante cuya pasión pone en su cara "expresión de diosa", y la intensidad suficiente para que la propia Catalina envidiara "su estado de gracia" (109). Es la emancipación emocional, sin duda alguna, la que logra romper con el modelo, y promover en la protagonista la "desacralización" del general: ella, que "oía sus instrucciones como un Dios" (24) al comienzo de la novela, le da muerte imaginaria cuando "contemplando al oso dormir" comprende que "yolo quise, yo hasta crế que nadie era más guapo, ni más listo ni más simpático, ni más valiente que él", pero "ya no" (224), "fingi que sacaba de mi cinto un puñal y se lo enterraba de últimas, antes de irme" (225). Sería interesante penetrar en el profundo simbolismo de esta escena, pero bástenos ahora recordar lo que Cirlot apunta con respecto al instrumento que maneja esta mujer: "Por la posibilidad de ser escondido -dice-el puñal simboliza el anhelo de agresión, la amenaza informulada, inconsciente. Servidor del instinto en la misma medida que la espada del espíritu, el puñal denota, con su tamaño, lo 'corto' del poder agresor, la carencia de ... potestad superior" (377). Este ficticio asesinato que da salida a lo inconsciente, nótese bien, aparece en un momento muy significativo: enamorada intensamente de Carlos, comprende de inmediato la falacia del contacto sexual con su marido: "lo dejé hacer. Pensé en Pepa diciendo: En el matrimonio hay un momento en que tienes que cerrar los ojos y rezar un Ave María. Cerré los ojos y me puse a recordar el campo" (223). No es solamente, por tanto, un anhelo de agresión social -aunque de estos elementos venga ciertamente acompañado-, el que reclama ese gesto imaginario de Catalina sobre el viejo general, sino el anhelo de su propia e intensa plenitud emocional. No un gesto que agrede a las múltiples convenciones cotidianas que representa la mujer, sino el que rasga carne y vestiduras para encontrar su centro, su intimidad. Es por esta obsesionada búsqueda de la individualidad femenina por la que la autora mexicana logra un discurso narrativo que haría, sin duda, las mieles de cualquier "posmodernista", al desarrollar su planteamiento novelesco 
dede una doble y nutrida "periferia": la perspectiva femenina (en relación a la hegemonía homocentrista) y la visión desde la intimidad (en relación a toda hegemonía social).

Cabría preguntarse aquí si en esa doble descentralización de las hegemonías no tiene la segunda un sentido quizá más corrosivo y generalizado: en un siglo de preferencias cientifistas, de mirada racional y relación utilitaria, la visión afectiva desde la intimidad, -y sus derivaciones ("lo mujer", "lo sensiblero", según lo conciben los ojos de Andrés)-, afecta y determina no tanto a un grupo social como a una conducta nueva y específica ( "la posmodernidad"?). Nada más periférico, según esta última interpretación, nada mayormente marginal que la revelación de lo íntimo, la aceptación de las pasiones, el desarrollo pleno de la subjetividad, la reconciliación, en suma, con una realidad que no es sólo tangible, expresados a veces con los "fragmentos" y "despojos" de la cultura oficialista. Esta contemplación periférica arremete así contra un caudal de tradiciones, y sobre todo de prejuicios, cuya base social y cultural es bien pal pable, y ante la que Angeles Mastretta despliega su estrategia: el discurso de la sinceridad. Tanto el lenguaje espontáneo, coloquial y hasta perverso (en cuanto que desnuda en seguida el carácter del hablante), la simplicidad de la estructura narrativa cercana en ocasiones a modelos de amplio eco y difusión popular, comolas referencias contra-culturales que salpican el texto, y a las que ya nos referimos en el anterior apartado, son elementos eficaces de un código periférico cuyo objeto es, precisamente, la diferencia, los márgenes de la otredad. De modoinevitable, como revelaba Antonio Skármeta, el acercamiento a la realidad "con la obsesión de un miope" que practican los nuevos narradores, no puede dejar atrás los innumerables motivos de la perturbación social, y los grados en que ésta se vive y se consuma. Frente a una literatura que olvidó la carne de la cotidianeidad, otra que se dirige sin rodeos a los espacios de ese olvido; frente a la hegemonía y la frialdad que Andrés desempeña hasta el final en la novela, la voz sincera e íntima de una mujer que digiere "contra-cultura" y "periferia", sabiduría "cotidiana" y "popular", en la medida en que no logra satisfacerse en un vientre oficialista (la gitana del barrio de la Luz no es sino el irónico recurso que opera contra determinados silencios de sello homocéntrico, "oficial": "¿Por qué no me enseñas? -le dije. -iA qué? -Pues a sentir. -Eso no se enseña, se aprende -contestón (13)). Clichés cuya inmediatez y popularidad, si se quiere, pudieran parecer lugares sospechosos, muy comunes, si no se profundiza lo bastante en un discurso cuyo interés reside en el uso irónico, y en la aceptación, de estos materiales de digestión difícil para cualquier hegemonia cultural.

La valoración final de Arráncame la vida nos permite, pues, sintetizar en el triple eje de "hegemonia, periferia y sinceridad" los mecanismos sustanciales de la obra de Angeles Mastretta, y entrever en ella algunos gestos que se han incorporado de algún modo a ese cambiante "imaginario social" del que hablara Ruffinelli. Susceptibles ambas partes no ya a la vertiginosa sucesión de 
acontecimientos, sino más bien, según la visión "posmodernista", a una dinámica y contagiosa simultaneidad, es curioso observar de nuevo la equivalencia profunda entre la sociedad y su paradigma literario; si es cierto que el discurso narrativo más reciente ha sido alterado con la aparición de lo periférico fragmentos díscolos de una realidad que han conseguido trastornar el "sano" juicio-, no es menos cierto que el general de la novela contempla en Catalina la viva imagen de esta transformación iluminativa, paradójica y alterada: "Ah, las mujeres - se lamenta-. No cabe duda que ya no son las mismas. Algo las perturb6 $^{\prime 12}$ (218).

\section{BIBLIOGRAFIA CITADA}

Cirlot, Juan Eduardo, Diccionario de símbolos, Barcelona: Labor, 1988, $7^{\mathrm{a}} \mathrm{ed}$. Domínguez Michael, Christopher, Antología de la narrativa mexicana del siglo $X X$, México: F.C.E., 1991.

Edwards, Jorge, "Situación actual de la novela de América Latina. La continuidad, la revisión, el cambio", Insula 512-513 (1989), 68.

Giardinelli, Mempo, "Panorama de la narrativa mexicana en los años 80 ", Insula 512-513 (1989), 22-25.

Mastretta, Angeles Arráncame la vida, Madrid: Alfaguara, 1987.

Morello-Frosch, Marta "Discurso erótico y escritura femenina", Escritura y sexualidad en la literatura hispanoamericana, Madrid: Fundamentos, 1990, 21-30.

Puleo, Alicia, "Perspectivas antropológicas de un problema de crítica literaria", Escritura y sexualidad en la literatura hispanoamericana, Madrid: Fundamentos, 1990, 7-19.

Ruffinelli, Jorge, “Los 80 ¿ingreso a la posmodernidad?”, Nuevo Texto Crítico 6 (1990), 31-42.

Sánchez Vázquez, Adolfo, "Radiografía del posmodernismo”, Nuevo Texto Crítico $6(1990), 5-15$.

${ }^{12}$ El subrayado es nuestro. 
